\section{FAMILY PRESENCE DURING RESUSCITATION - A PROTOCOL OF A COCHRANE SYSTEMATIC REVIEW}

\begin{abstract}
1,2,3 MA Rubin*, ${ }^{4}$ SF Herling, ${ }^{5}$ TW Klausen, ${ }^{6,7,8} \mathrm{P}$ Jabre, ${ }^{1,2,3} \mathrm{AM}$ Møller. ${ }^{1}$ Department of Anaesthesia, Herlev and Gentofte Hospital, University of Copenhagen, Herlev, Denmark; ${ }^{2}$ Cochrane Emergency and Critical Care, Herlev and Gentofte Hospital, University of Copenhagen, Herlev, Denmark; ${ }^{3}$ Cochrane Anaesthesia, Herlev and Gentofte Hospital, University of Copenhagen, Herlev, Denmark; ${ }^{4}$ The Neuroscience Centre, Rigshospitalet, University of Copenhagen, Copenhagen, Denmark; ${ }^{5}$ Department of Hematology, Herlev Hospital, Herlev, Denmark; ${ }^{6}$ Assistance Publique-Hôpitaux de Paris (AP-HP), SAMU (Service d'Aide Médicale Urgente) de Paris, Hôpital Universitaire Necker-Enfants Malades, Paris, France; 'Institut national de la santé et de la recherche médicale (Inserm) U970, ParisCentre de recherche Cardiovasculaire (PARCC), Université Paris Descartes, Paris, France; ${ }^{8}$ Cochrane Pre-hospital and Emergency Care Field, Paris, France
\end{abstract}

\subsection{6/bmjopen-2019-EMS.39}

Background Family presence during resuscitation (FPDR) is a growing praxis despite most evidence is of low quality. ${ }^{1}$ The aim of this review is to investigate if FPDR might decrease PTSD-related symptoms including signs of depression/anxiety. We also want to explore the effect on medical care and patient survival.

Method We will include randomized controlled trials (RCTs) and perform a meta-analysis after assessment of heterogeneity, if appropriate. For dichotomous outcomes Mantel Haenszel test will be used, for continuous outcomes inverse variance and for rare events, Peto's odds ratio. The quality of evidence will be assessed using the GRADE-method.

Results Preliminary search results identified 564 articles for screening leading to four RCTs. This leads to a sample size of 1388. A cluster-RCT of 570 relatives showed positive effects of FPDR on relatives' psychological outcomes during pre-hospital CPR and no effects on patient mortality or personnel distress. ${ }^{2}$ The largest RCT with a sample size of 705 investigated FPDR of paediatric patients, but had a high risk of bias. $^{3}$

Conclusion We hypothesize that there is a lack of high quality research regarding FPDR, but that the evidence indicate an association with overall positive results on relatives' psychological outcomes and that it doesn't affect the healthcare professionals, morbidity, or mortality of the patients negatively.

\section{REFERENCES}

1. Oczkowski SJ, Mazzetti I, Cupido C, Fox-Robichaud AE. The offering of family presence during resuscitation: a systematic review and meta-analysis. Journal of intensive care 2015:3:41.

2. Jabre P, Belpomme V, Azoulay E, Jacob L, Bertrand L, Lapostolle F, et al. Family presence during cardiopulmonary resuscitation. The New England journal of medicine 2013;368(11):1008-18.

3. Dudley NC, Hansen KW, Furnival RA, Donaldson AE, Van Wagenen KL, Scaife ER. The effect of family presence on the efficiency of pediatric trauma resuscitations. Annals of emergency medicine 2009;53(6):777-84.e3.

Conflict of interest P. Jabre is the first author of an included primary study (which she has conducted) in the Cochrane Review (2). She will not extract data from her own study. Instead, M.A. Rubin and A.M. Møller will extract these data, and check the interpretation against the study report and any available study registration details or protocol.

Funding None.

\section{LONG AMBULANCE RESPONSE TIME IS ASSOCIATED WITH HIGHER INCIDENCE OF CARDIOPULMONARY RESUSCITATION AND DEFIBRILLATION BY DISPATCHED CITIZEN FIRST-RESPONDERS}

${ }^{1} \mathrm{~L}$ Andelius ${ }^{*},{ }^{1,3} \mathrm{CM}$ Hansen, ${ }^{1} \mathrm{~F}$ Lippert, ${ }^{1,2} \mathrm{~L}$ Karlsson, ${ }^{4} \mathrm{C}$ Torp-Pedersen, ${ }^{2,5} \mathrm{GH}$ Gislason,

${ }^{1,2} \mathrm{~F}$ Folke. ${ }^{1}$ Emergency Medical Services Copenhagen, University of Copenhagen, Denmark; ${ }^{2}$ Department of Cardiology, Herlev Gentofte University Hospital, Denmark; ${ }^{3}$ Department of Cardiology, Nephrology, and Endocrinology, Copenhagen University Hospital Hillerød, The Region of Northern Zealand, Denmark; ${ }^{4}$ The Department of Health Science and Technology, Aalborg University, Aalborg, Denmark; ${ }^{5}$ The Danish Heart Foundation, Copenhagen, Denmark

\subsection{6/bmjopen-2019-EMS.40}

Background Dispatching citizen first-responders by a smartphone application (app) might increase incidence of bystander cardiopulmonary resuscitation (CPR) and defibrillation in outof-hospital cardiac arrest (OHCA). We investigated citizen first-responder rates of $\mathrm{CPR}$ and defibrillation according to ambulance response time.

Method From September 1, 2017 to September 1, 2018, 23 021 citizen first-responders registered through the app. After being dispatched, citizen first-responders reported whether they performed CPR or defibrillation. Distances were calculated as straight line. Ambulance response time was defined as time from dispatch to vehicle stop. Citizen first-responder CPR and defibrillation were calculated according to ambulance response times (10 $\mathrm{min})$.

Results The Emergency Dispatch Center activated the system in 837 suspected OHCAs where 51\% $(n=431)$ were confirmed as cardiac arrests eligible for inclusion. The median distance from citizen first-responder to OHCA was 628 meters (365,902; Q1, Q3), corresponding to $5 \mathrm{~min}$ and $14 \mathrm{~s}$ citizen first-responder response time (assumed speed of $2 \mathrm{~m} / \mathrm{s}$ ). For ambulance response times $10 \mathrm{~min}$, citizen first-responders performed CPR in $29 \%(n=42), 40 \%(n=91)$, and $65 \%(n=39)$ of the cases, $\mathrm{p}<0.0001$, respectively. The corresponding figures for defibrillation according to ambulance response time were $8 \%(n=11)$, $16 \%(n=36)$, and $28 \%(n=17), p=0.0005$. Citizen first-responders defibrillated at total of $15 \%(n=64)$ of all OHCAs.

Conclusion A total of $15 \%$ of all OHCA patients were defibrillated by citizen first-responders. Longer ambulance response times were associated with higher rates of citizen first-responder CPR and defibrillation.

Conflict of interest Dr. CM. Hansen, Dr. F.Folke and Dr. F.Lippert have received research grants from the Lærdal Foundation. None of the other authors reported conflict of interest.

Funding Dr. L. Andelius, Dr. L. Karlsson, Dr. CM. Hansen and Dr. F.Folke have received unrestricted funding from the Danish Foundation TrygFonden.

\section{NATIONAL EARLY WARNING SCORE (NEWS) EVALUATION IN AN AMBULANCE-NURSE: ONE-YEAR EXPERIENCE IN PORTUGAL}

${ }^{1} \mathrm{P}$ Vasconcelos ${ }^{*},{ }^{1} \mathrm{~A}$ Oliveira, ${ }^{1} \mathrm{~T}$ Augusto, ${ }^{1} \mathrm{~L}$ Ladeira, ${ }^{1} \mathrm{~J}$ Lourenço, ${ }^{2} \mathrm{~F}$ Barros, ${ }^{2} \mathrm{R}$ Ramos. ${ }^{1}$ Registered Nurse at National Institute of Emergency Medicine, Portugal; ${ }^{2}$ Medical Doctor at National Institute of Emergency Medicine, Portugal

10.1136/bmjopen-2019-EMS.41 
Background Several studies published showed the application of the Early Warning Scores (EWS) in prehospital setting. This article aims to reflect the prehospital emergency nurse's intervention, acting under pre-established medical protocols, in the clinical evolution of the critically ill patient, through the application of the NEWS2 score.

Method This is a retrospective analysis of cases during one year in an urban ambulance-nurse staffed (SIV-Lisboa), by the application of the NEWS2 in three different moments: at arrival of the team, after the first interventions and at hospital admission.

Results Between October 1, 2017 and September 30, 2018, SIV-Lisboa assisted 2387 patients, of which 1825 patients were elected to this study. At the arrival of the ambulance team, $36.77 \%$ of the patients showed Red Score, $0.55 \%$ High Score, 1.32\% Medium Score, 60\% Low Score and in $1.37 \%$ of the cases there were a missing item. At hospital admission, $52.9 \%$ of the red scored patients improved to lower scores.

Conclusion The application of NEWS2 by an ambulance-nurse staffed allows to identify the criticity of the patient, optimizing the communication between nurse and doctor, in dispatch center, allowing to evaluate the success of the interventions performed by nurses in the prehospital phase. The analysis of the data concluded that the nursing intervention proved to be effective in stabilizing the critical patient. In the future, it will be interesting to expand the application of this score to all ambulance-nurse staffed, making possible a national wide analysis.

\section{REFERENCES}

1. Morgan $R$, Williams $F$, Wright $M$. An early warning scoring system for detecting developing critical illness. Clin Intensive Care. 1997; 8-100.

2. Martín-Rodrigues F, Castro-Villamor MA, Del Pozo Vegas C, Martin Conti JL, Mayo-Iscar A, Delgado Benito JF, Del Brio Ibañez P, Arnillas-Gomez P, EscuderoQuadrillero C, Lopez-Izquierdo R. Analysis of the early warning score to detect critical or high-risk patients in the prehospital setting. Intern Emerg Med. 2019 Jan.

3. Shaw J, Fothergill R, Clarck S, Moore F. Can the prehospital National Early Warning Score identify patients most at risk from subsequent deterioration? BMJ 2017.

Conflict of interest None.

Funding None. PRODUCES HYPERKALEMIA?

L Jimenez*, C Sanchez, M Castillo, E Torres, C Escudero, J Hortelano.

\subsection{6/bmjopen-2019-EMS.42}

Background Succinhylcholine is a depolarizing relaxant that can cause hyperkalemia. Some clinical studies have shown serious hyperkalemia cases subsequent to the administration of succinhylcholine, reason why many Outpatient Services have withdrawn succinhylcoline from their therapeutic arsenal, and therefore the possibility to perform a Rapid Sequence Intubation (RSI).

Method Our hypothesis is that succinhylcholine, when used as a unique dose in RSI, can cause mild hyperkalemia, as observed in a retrospective observational study of SAMUR-PC assisted cases in 2017. This study includes patients above 18 . RSI had been performed in all cases - regardless of their pathologies, and pothasium levels had been measured before and after the administration of succinhylcholine.

Results This study includes patients above 18. RSI had been performed in all cases - regardless of their pathologies, and pothasium levels had been measured before and after the administration of succinhylcholine. A sample size (n) was obtained in 95 cases. The average pothasium before RSI was 4. 0049 with a standard deviation of 0.9022 while the average pothasium level after RSI was 4.0758 with a standard deviation of 0.7807 . In order to compare the mid-range of both tests and determine significant differences, we performed the Wilconson $\mathrm{T}$ test, which shows that there is no statistical significance between the pothasium averages before and after the administration of succinhylcholine.

Conclusion It is therefore confirmed for the first time in the outpatient environment that there is no significant increase of pothasium levels.

\section{REFERENCES}

1. Procedures manual of SAMUR-PC.

2. www.reanimacion.net.Succinylcholine intense debate about its contraindications.

3. www.medicineintensive.org. prevalence of Succinylcholine in intensive care units.

Conflict of interest None.

Funding None. 